\title{
Integrating multiple types of data to predict novel cell cycle-related genes
}

\author{
Lin Wang ${ }^{1}$, Lin Hou ${ }^{1,2}$, Minping Qian ${ }^{1,2}$, Fangting $\mathrm{Li}^{1,4^{*}}$, Minghua Deng ${ }^{1,2,3^{*}}$ \\ From The 4th International Conference on Computational Systems Biology (ISB 2010) \\ Suzhou, P. R. China. 9-11 September 2010
}

\begin{abstract}
Background: Cellular functions depend on genetic, physical and other types of interactions. As such, derived interaction networks can be utilized to discover novel genes involved in specific biological processes. Epistatic Miniarray Profile, or E-MAP, which is an experimental platform that measures genetic interactions on a genomewide scale, has successfully recovered known pathways and revealed novel protein complexes in Saccharomyces cerevisiae (budding yeast).

Results: By combining E-MAP data with co-expression data, we first predicted a potential cell cycle related gene set. Using Gene Ontology (GO) function annotation as a benchmark, we demonstrated that the prediction by combining microarray and E-MAP data is generally $>50 \%$ more accurate in identifying co-functional gene pairs than the prediction using either data source alone. We also used transcription factor (TF)-DNA binding data (Chipchip) and protein phosphorylation data to construct a local cell cycle regulation network based on potential cell cycle related gene set we predicted. Finally, based on the E-MAP screening with 48 cell cycle genes crossing 1536 library strains, we predicted four unknown genes (YPL158C, YPR174C, YJR054W, and YPR045C) as potential cell cycle genes, and analyzed them in detail.

Conclusion: By integrating E-MAP and DNA microarray data, potential cell cycle-related genes were detected in budding yeast. This integrative method significantly improves the reliability of identifying co-functional gene pairs. In addition, the reconstructed network sheds light on both the function of known and predicted genes in the cell cycle process. Finally, our strategy can be applied to other biological processes and species, given the availability of relevant data.
\end{abstract}

\section{Background}

According to [1], "mutations in two genes produce a phenotype that is surprising in light of each mutation's individual effects. This phenomenon, which defines genetic interaction, can reveal functional relationships between genes and pathways." Thus, deciphering genetic interaction networks via high-throughput technologies can both reveal the schematic wiring of biological processes and predict novel genes. Recently, several such high-throughput technologies have been developed to identify genetic interactions at the genome scale, including Synthetic Genetic Array (SGA) [2], Diploid-based

\footnotetext{
*Correspondence: li_fangting@pku.edu.cn; dengmh@pku.edu.cn

${ }^{1}$ Center for Theoretical Biology, Peking University, Beijing 100871, China Full list of author information is available at the end of the article
}

Synthetic Lethality Analysis on Microarrays (dSLAM) [3], and Epistatic Miniarray Profile (E-MAP) [4]. The first two approaches aim to identify synthetic lethal, or negative, interactions, meaning that the double mutant is more lethal than the corresponding single mutants. On the other hand, assuming that the expected phenotype of a double mutation reflects the additional effects of the single mutations, E-MAP, an extension of SGA, gains power by identifying positive as well as negative interactions, which, in this case, would indicate that the double mutant is healthier than expected.

Here, we exploited the E-MAP methodology to discover novel genes involved in the cell cycle process in budding yeast. The distinct advantage of using E-MAP is the potential of discovering functionally associated
C Biomed Central

(c) 2011 Wang et al; licensee BioMed Central Ltd. This is an open access article distributed under the terms of the Creative Commons Attribution License (http://creativecommons.org/licenses/by/2.0), which permits unrestricted use, distribution, and reproduction in any medium, provided the original work is properly cited. 
genes which do not otherwise physically interact. Physical interaction assays, such as the yeast two-hybrid system or DNA-binding microarrays, are unlikely to reveal these associations. Despite the superiority of E-MAP, interpretation of the data is still challenging. First, genetic interactions occur both between and within functional modules. Thus, the function of a gene cannot be determined by its interacting partners. Second, EMAP suffers from high false positive and negative rates, making it difficult to infer genetic interaction accurately and sufficiently. Consequently, the integration of external information, such as gene expression, Transcription Factor (TF)-DNA binding (chip-chip) and protein phosphorylation, is necessary in order to identify novel genes involved in the cell cycle process.

Several methods have been developed to integrate multiple types of data to infer a transcription regulatory network in eQTL analysis, including mRNA expression, chip-chip, physical interaction and protein phosphorylation [5-8]. In this paper, we integrated genetic interaction and other genomic data to construct a specific network which we then applied to the cell cycle process in budding yeast.

\section{Results}

\section{Construct a potential cell cycle-related gene set}

As indicated in Figure 1, our strategy, which integrates multiple types of data, aims to include all potential cell cycle genes within the known cell cycle gene set. Since both genetic interacting and co-expressed gene pairs tend to be co-functional, we hypothesized that a potential cell cycle related gene set with higher confidence can be achieved through combining the two data sources, compared with using either data alone.

To accomplish this, we first quantitatively measure whether genetic interactions and co-expression indicate co-functional membership and, if so, to what degree. The E-MAP method was adopted for genetic interaction analysis. Forty-eight known cell-cycle genes (KCCGs, Table S1 in Additional file 1) were screened against a library of 1536 test strains in budding yeast, yielding a quantitative value (S-Score defined in [3]) for 67680 gene pairs (91\% of all possible pair-wise measurements). Co-expressed data were then calculated from 8 groups of time-course expression datasets generated in four previously published studies (see Methods and materials for details). To calculate the enrichment of co-functional gene pairs over random gene pairs, we first compute the fraction $\mathrm{f}$ of interactions at each $\mathrm{S}$-score (or cc, correlation coefficient of expression) (Figure 2A and 2B) or simultaneously more extreme than $\mathrm{s}$ and $\mathrm{cc}$ (Figure $2 \mathrm{C}$ and 2D) that fall inside one biological process term in GO for certain bin sizes. Then the enrichment is the ratio $f / r$, where $r$ is the fraction of random gene pairs which participate in the same GO biological processes.

As expected, Figure 2A confirms that gene pairs having a higher cc are more likely to be co-functional. Also, Figure 2B shows that gene pairs with both significant positive and negative S-scores are more likely to be cofunctional. By comparing the enrichment between Figures $2 \mathrm{~A}$ and $2 \mathrm{~B}$, it is apparent that extreme S-scores could indicate co-functional membership more efficiently.

When combining these two kinds of data, we found that they were complementary. As shown in Figure 2C, for a certain cut-off of S-scores, gene pairs with a higher correlation of expression are more likely to be co-functional, and vice versa. Therefore, the results approved our original hypothesis that combining these two kinds of information could help to construct a more accurate potential cell cycle related gene set. We adopted an area by which to define significantly interacting gene pairs based on the data in Figure 2C. For a positive genetic interaction area, we require that the enrichment over random be larger than 2 , and for a negative genetic interaction area, we require that it be larger than 4 . Then the constraints are (S-score $>2.5$ and cc $>0.9$ ) or Sscore $>6$ or $(\mathrm{S}$-score $<-3$ and $\mathrm{cc}>0.9)$ or $(\mathrm{S}$-Score $<-14$ and cc $>0.85)$. Compared to the most powerful method at each point, the combination is generally $>50 \%$ more accurate in the areas defined above (Figure 2D). Finally, 259 gene pairs between 206/1536 test strains and 48 KCCGs passed the filter. We use these 206 test genes as the potential set of cell cycle-related genes (PCCGs).

\section{Recovery of known genetic interactions with our E-MAP}

We compared our E-MAP data with the benchmark data. Similar to previous work [9], we tested the sensitivity and precision of the E-MAP data (see Methods and materials). Compared to genetic interactions in BIOGRID, both the positive and negative interactions are very precise $\left(\mathrm{p}\right.$-value $\left.<10^{-50}\right)$.

We also tested the efficiency when combining E-MAP with DNA microarray data. When the co-expression test was applied, the significance level of precision increases around 2-fold (Table S2 in Additional file 2). This result indicates that co-expression does indeed provide extra information about genetic interaction. Hence, our strategy can be used to identify potential cell cycle genes and their relationships with known cell cycle genes, thus enabling us to construct a reliable network.

We also compared our S-score with previously published large-scale genetic interaction data [8]. Significantly interacting gene pairs show obvious correlation between the two datasets $(r=0.64$, Figure S1 in Additional file 3). 


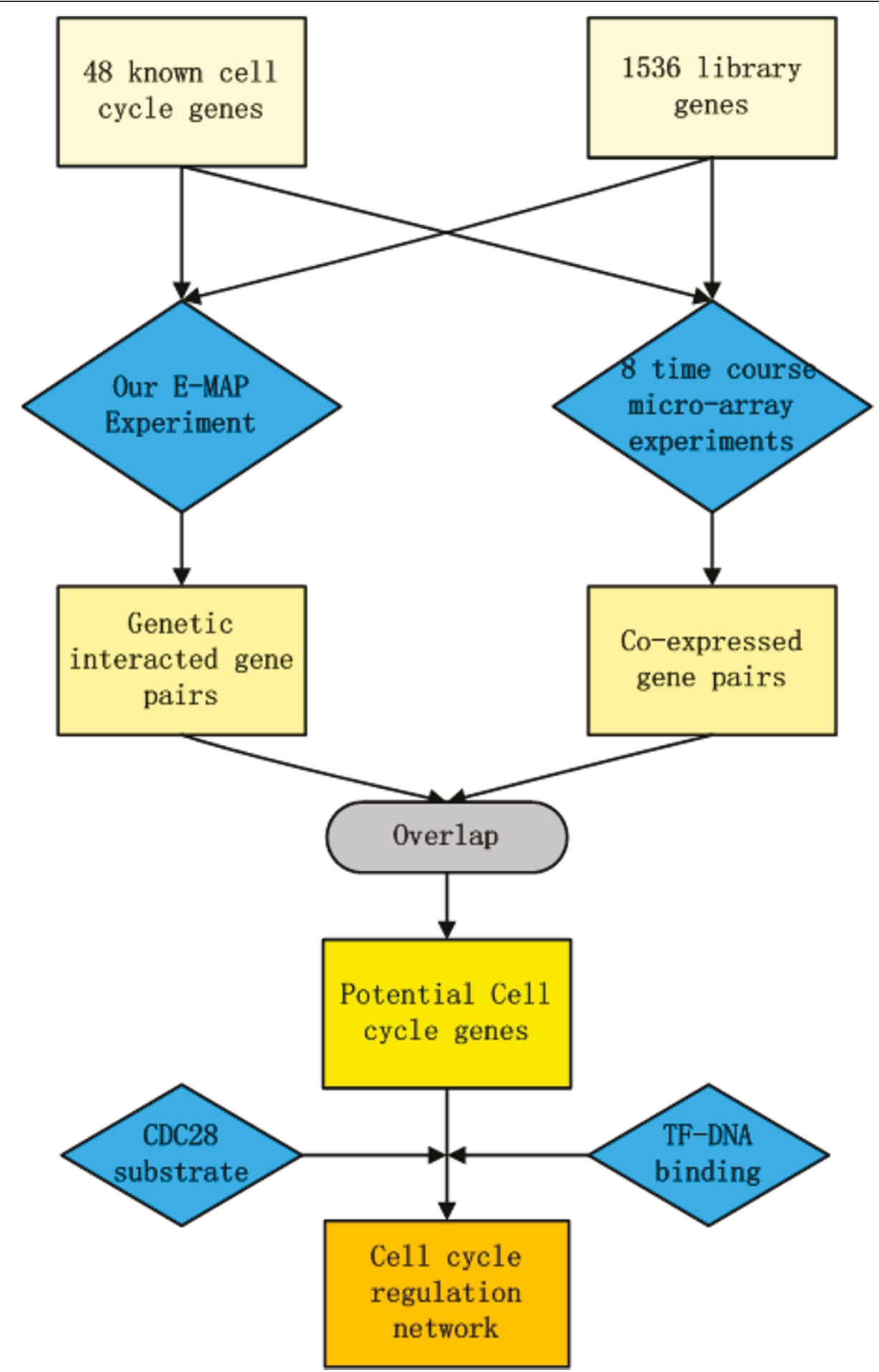

Figure 1 Overview of our strategy to integrate multiple types of data to identify cell cycle gene and infer regulatory pathways.

\section{Functional enrichment analysis in the PCCGs}

Functional enrichment analysis was performed on all GO biological process terms in both positive and negative parts of PCCGs. We defined the positive part as those genes having ( $\mathrm{S}$-score $>2.5$ and $\mathrm{cc}>0.9$ ) or $\mathrm{S}$ - score $>6$ and the negative part as those genes having $(\mathrm{S}-\mathrm{score}<-3$ and $\mathrm{cc}>0.9)$ or $(\mathrm{S}-\mathrm{Score}<-14$ and $\mathrm{cc}>0.85)$. We distinguished these two parts because the principles on which positive and negative genetic interaction are based may be different for functional analysis, as 

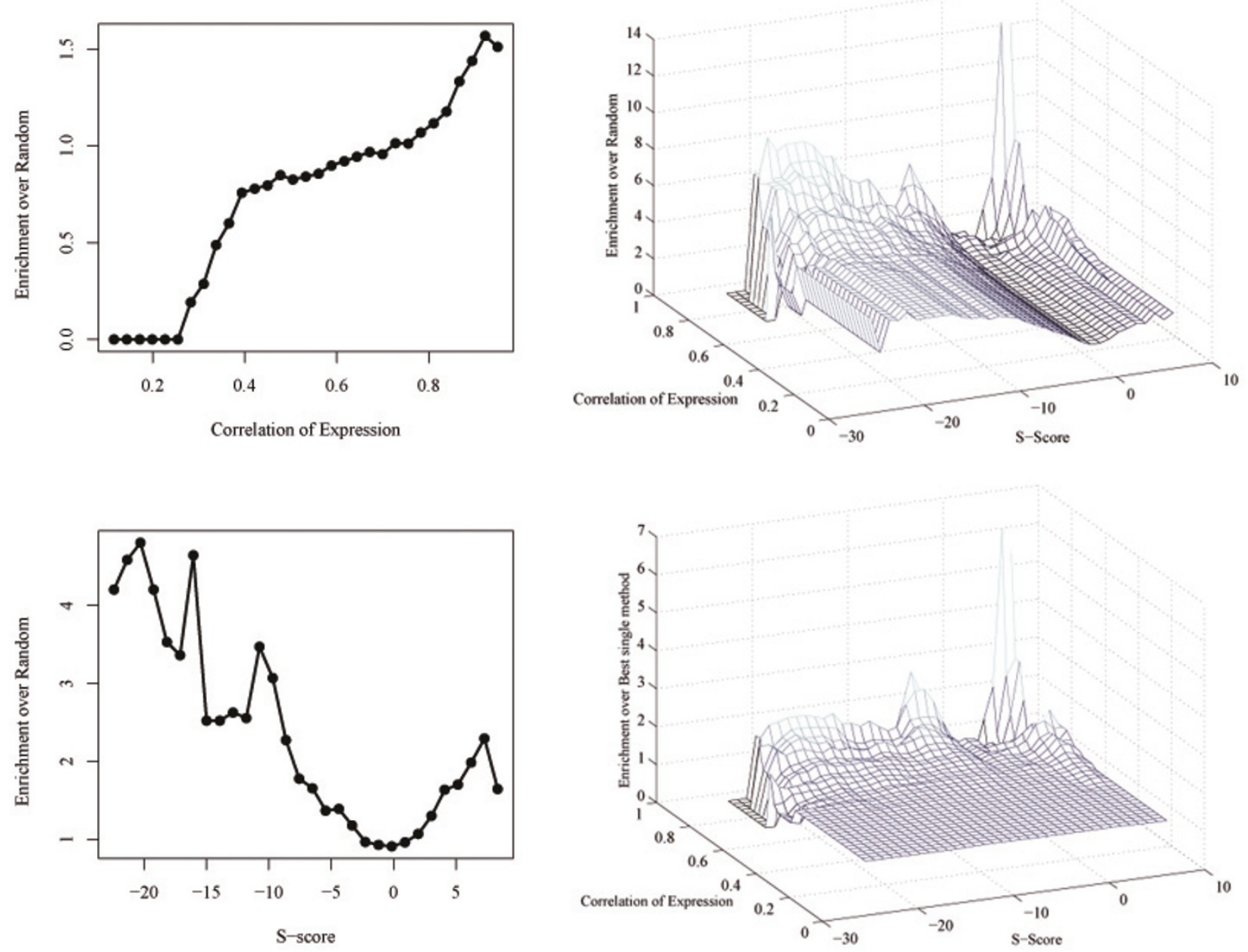

Figure 2 Combining genetic interaction and co-expression data to define co-functional gene pairs. High correlation coefficient (A) and extreme S-Score both correspond with known co-functional gene pairs in Gene Ontology (GO) (bin size is 1 Correlation and 1.5 for S-Score). (C) Gene pairs have more extreme S-score and larger correlation of expression have higher enrichment. (D) When combining two criteria, the enrichment is significantly improved in extreme S-score and large correlation area.

discussed in a previous study [10]. For the positive part, only one functional category, "nucleosome organization," was enriched under $98 \%$ confidence level $(\mathrm{q}=0.012)$. For the negative part, five functional categories were enriched, including "DNA-dependent DNA replication," "chromatin assembly," "interphase of mitotic cell cycle," "cell cycle checkpoint" and "regulation of organelle organization" ( $q=0.014,0.013,0.015,0.009,0.012)$. All these biological processes can either be interpreted as related to the cell cycle process, or just part of it. In addition, KCCGs were found to be mainly involved in "regulation of organelle organization," "regulation of mitotic cell cycle," "interphase of mitotic cell cycle," "regulation of cell cycle process" and "cell cycle checkpoint" processes. Hence, in the negative part, there are more directly cofunctional genes than the positive part. This is consistent with the surprising conclusion of previous work [9] indicating that negative, in contrast to positive, genetic interactions always occur between genes with overlapping functions.

Finally, we also analyzed the functional enrichment of all PCCGs. Three functions, including "chromatin assembly," "regulation of organelle organization" and "nucleosome organization," were enriched ( $q=0.015$, $0.009,0.002)$. This suggests the importance of separating PCCGs into two parts for a functional enrichment analysis. Such separation further helps us to understand how known cell cycle genes, both positive and negative, interact in terms of their functions and also helps us to find specific functions only enriched in one of the two parts, such as "DNA-dependent DNA replication," "interphase of mitotic cell cycle" and "cell cycle 
checkpoint." which only enriched in negative part but not in all PCCGs.

\section{A cell-cycle transcriptional network based on the PCCGs and KCCGs}

In the next step, we searched for main transcription factors (TFs) regulating both the PCCGs and the KCCGs based on TF-DNA binding data (Chip-Chip), and then we constructed the resulting transcriptional regulatory network. In previous studies, Chip-Chip data are usually combined with expression information to construct the regulatory network. In our method, periodic expression was required for TF inclusion. Since most genes involved in the cell cycle process are expressed periodically, it is reasonable to assume corresponding periodicity of their transcriptional regulators. In addition, we also assumed that the regulatory targets of a TF involved in the cell cycle would be enriched for the known cell cycle gene. Hence, our transcription network was based on TFs enriched for cell cycle targets for both potential and known cell cycle genes combined from the pool of PCCGs and KCCGs. The significance of periodicity and enrichment of cell cycle genes can be calculated (see Methods and materials). Both approaches tend to select TFs which are known to be involved in cell cycle regulation according to MIPS functional annotation (Figure 3[11]).

Periodicity and enrichment are consistent criteria since most of the known cell cycle TFs rank at the top in both cases. However, some TFs are ranked differently (See Table S3 in Additional file 4). For example, $M c m 1$ is ranked 6/130 in the enrichment test (ET); however, it ranks 124/183 in the periodic test (PT), which means that its expression does not show periodicity. We know that Mcm1 regulates different phases during the cell cycle $[12,13]$, and its expression will not be periodic. However, many of its neighboring genes in the transcriptional network are cell cycle genes, making its identification possible in the enrichment test. Similar to Mcm1, Skn7 ranks 23 and 114, respectively, in ET and PT. In contrast, HCM1 is ranked 3 in PT, but 42 in ET. One possible explanation of this apparent difference is that the PCCGs and KCCGs only cover a limited part of cell cycle-related genes, and some targets of HCM1 are missing in this set. Other examples like YHP1 are similar to HCM1. Based on this analysis, a TF that is significant in either test should be included. Hence, we use the multiplication of the two ranks as an index, and we use its rank to evaluate the priority order (see Methods and materials for details).

To determine how many TFs should be involved, we examined the coverage rates of TFs. The coverage rate is evaluated at two levels: the fraction of genes which are targets of the selected TFs in the PCCGs and KCCGs and the fraction of gene pairs which are co-regulated by any one of the selected TFs. In the PCCGs and KCCGs, 232/
236 genes are involved in the chip-chip dataset (at least one TF can bind to them), and 77 gene pairs, which are both genetically interacting and co-expressed, can be simultaneously bound by the same TF. We noticed that when the top 25 TFs are selected, most of the 232 genes and 77 gene pairs (75\% and 97\%, respectively) could be covered (Figure S2 in Additional file 5). The cover rate increases quite slowly when more TFs are selected. We therefore used these 25 TFs to construct the transcriptional network based on the PCCGs and KCCGs.

\section{Enrichment for cell-cycle genes and TFs}

We next determined whether the PCCGs are enriched with known cell-cycle genes. Among the PCCGs, we calculated the proportion of genes which are annotated to participate in the cell cycle process (in MIPS database) and used the hyper-geometric distribution to define the p-values. About $1 / 2$ of the PCCGs (94/206) were determined to be known cell-cycle and DNA processing genes $\left(p=6 \times 10^{-6}\right)$. We performed the same test to the selected TFs. Eighteen of them are known to be cell cycle TFs $\left(p=5 \times 10^{-11}\right.$, Table S4 in Additional file 6).

\section{Enrichment for $C D C 28$ substrates}

Since cell cycle events are controlled by cyclin-dependent kinases (CDKs), we investigated whether Cdk1 $(C D C 28)$ substrates were enriched in our PCCGs and selected TFs. As expected, both of them turned out to be enriched with $C D C 28$ substrates (Table S5 in Additional file 7), further supporting the finding that both PCCGs and selected TFs are cell cycle-related.

\section{Formation of a cooperative transcriptional network by selected TFs is supported by indirect evidence}

We compared the difference between using all TFs in the database and only the selected 25 TFs to explain indirect transcriptional relationships between the 25 TFs and 232 target genes. Based on comparing the wild-type and TF mutant microarray data, we could tell how one TF could affect the expression of each gene. This data reflects the transcriptional relationship between the TFs and targets although the relation could be indirect. This independent evidence, which describes the transcriptional network, can be utilized to validate the network we constructed.

Between our 25 TFs and the 232 target genes, there are 140 indirect TF-target pairs. By using the transcriptional relationships of all 183 TFs in the chip-chip dataset, 103/140 pairs could be connected within three steps, although for more steps, quite a few indirect pairs could be explained (Figure S3 in Additional file 8). We also tested the fraction of indirect TF-target pairs which could be connected by only using the relationships of the 25 TFs. The result (Figure S3) shows that the sub TF-target network can explain $85.4 \%(88 / 103)$ of the 


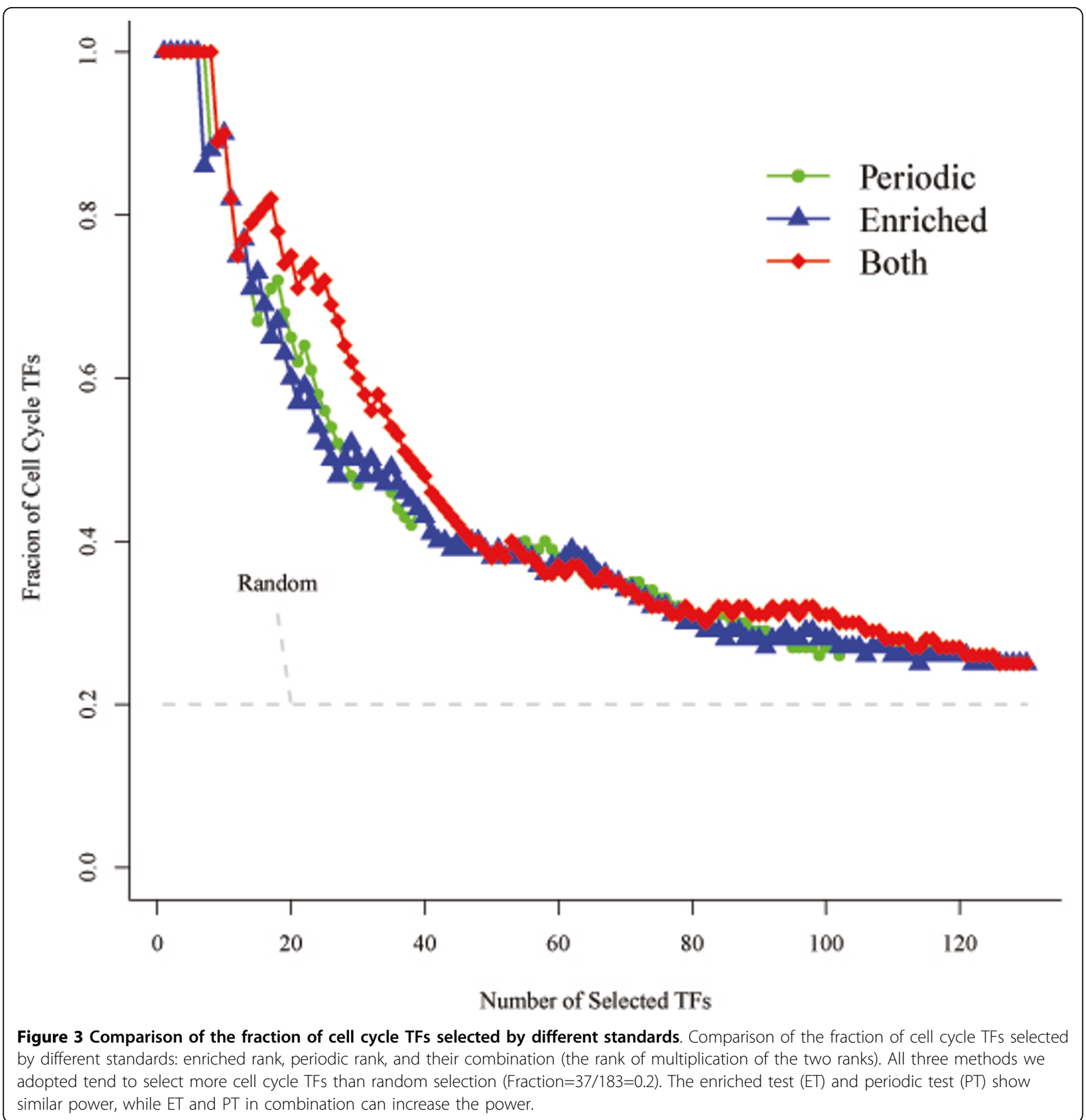

indirect relations in the first three steps. This result illustrates that the 25 TFs form a cooperative transcriptional network which can explain its indirect connections quite well.

\section{Clustering of the constructed transcriptional network}

To understand the structure of our constructed transcriptional network, we used the transcriptional profile to do cluster analysis (Figure S4 in Additional file 9). That TFs with similar function regulate similar targets in the network can be inferred by the presence of several established cooperating TF clusters, such as Ace2/ Swi5, Fkh1/Fkh2/Mcm1 and Swi4/Swi6/Mbp1. In addition, genes with similar function can also cluster together and have meaningful explanation in the context of their TFs. Figure 4 shows three such functional clusters. The first one contains $\mathrm{G} 1 / \mathrm{S}$ and $\mathrm{S}$ phase functional genes which are simultaneously regulated by important G1/S transcriptional regulators, SBF (Swi4/Swi6) and MBF $(M b p 1 / S w i 6)$. The second contains DNA 


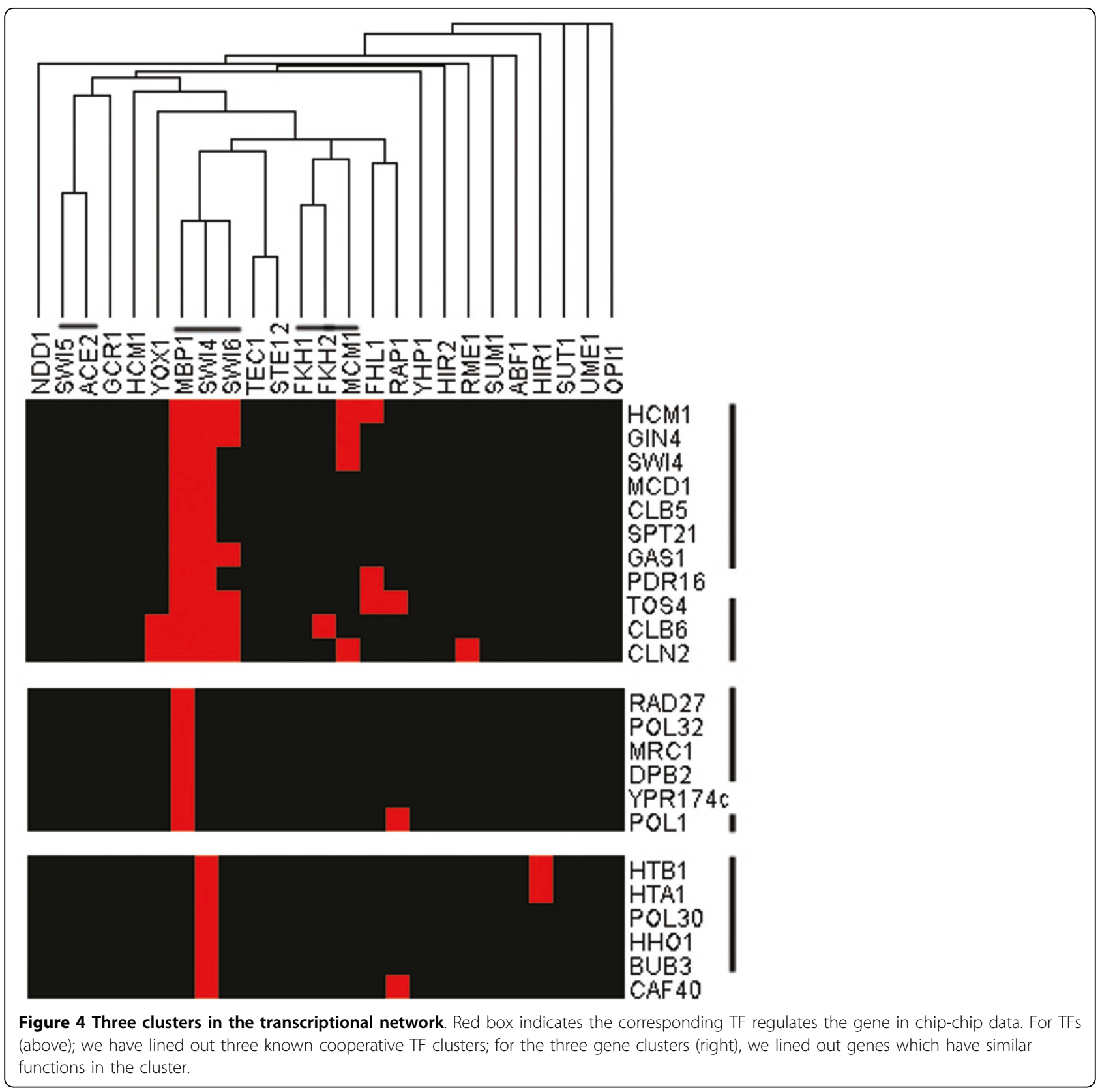

replication-related genes which are mainly regulated by Mbp1. The third contains chromosome segregation- and budding-related genes which are mainly regulated by Swit. These results are consistent with our knowledge about the function of SBF and MBF in the cell-cycle process. Hence, the structure of our transcriptional network revealed by the cluster analysis could also be used to infer functional relationships between genes.

\section{Potential cell cycle-related genes}

Based on E-MAP, expression, chip-chip, and protein phosphorelation data and the analysis above, we could identify PCCGs and know about its structure in transcriptional network (results are summarized in Table S6 in Additional file 10), Among the PCCGs, we will introduce four genes with unknown function (Figure 5).The first one is YPL158C, which genetically interacts with PCL9, AMN1 and BUD4. These four genes are all regulated by known TFs in $M$ phase (including G2/M and M/G1). The expression data show that YPL158C, PCL9 and $A M N 1$ are simultaneously expressed and that their peak value of expression is later than that of $A C E 2$ and $S W I 5$, which are their transcriptional regulators, as well as BUD4 (Figure 6A). This is consistent with the 


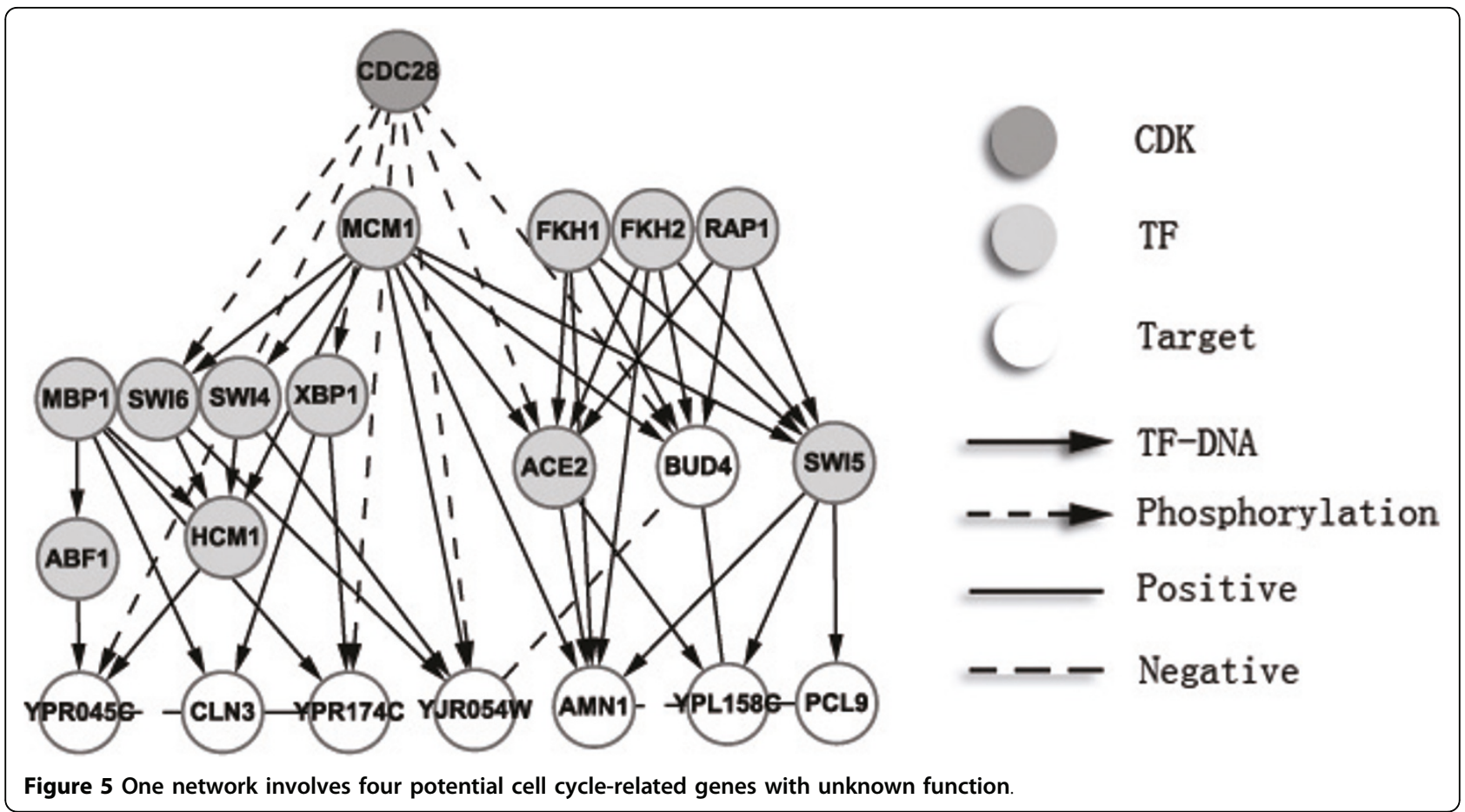

regulatory network because $B U D 4, A C E 2$, and $S W I 5$ are mainly regulated by $F K H 1 / 2$ and $M C M 1$, while $Y P L 158 C$ is mainly regulated by $A C E 2$ and SWI5. They all act in M phase or early G1 phase. Based on these observations, YPL158C is possibly involved in $\mathrm{M}$ phase and co-operates with PCL9, AMN1 and BUD4. The second is YPR174C, which genetically interacts with CLN3 and potential substrates of CDC28. YPR174C and CLN3 are co-regulated by $M B P 1$ and $X B P 1$, another known cell cycle TF, which was not selected in the procedure above because of low periodic rank (rank in PT: 120; rank in ET: 11; final rank: 34). According to the description in SGA, XBP1 is a member of the $S W I 4 / M B P 1$ family. Since $M B P 1$ and $X B P 1$ do not have significant periodic expression, we compared the expression of SWI4 with CLN3 and YPR174C. We found that SWI4 and $Y P R 174 C$ are significantly co-expressed and that CLN3 and YPR174C also show a co-expression pattern, but with two time points lagging (Figure 6B). It is convincing to consider that YPR174C might be involved in G1 phase in cell cycle process since all other related genes are mainly acting in this phase. In addition, based on the transcriptional analysis above, YPR174C is mainly regulated by $M B P 1$; hence, it is possibly involved in the DNA replication process. The third one is YJR054W, which genetically interacts with BUD4 and potential substrates of $C D C 28$. In Chip-Chip data, BUD4 is regulated by MCM1. YJR054W is regulated by SWI4 and $S W I 6$ which are also regulated by MCM1. In the expression data (Figure 6C), we found that the expressions of
SWI4 and BUD4 are highly negatively correlated. This can be explained by the fact that $M C M 1$ participates in the formation of both repressor and activator complexes, and SWI4 and BUD4 may be regulated by different complexes. The expression of YJR054W is similar to $S W I 4$ and slightly lags, which supports the regulation between them. Since BUD4 can influence the next round of budding and $S W I 4 / 6$ mainly regulates the G1 phase, YJR054W may be involved in M/G1 phase and may co-operate with BUD4. The last one is YPR045C, which genetically interacts with $C L N 3$, albeit negatively, and potential substrates of $C D C 28$. It is regulated by $H C M 1$ and $A B F 1$ which both regulate S-phase during the cell cycle process. YPRO45C is negatively correlated with HCM1 (Figure 6D), which may suggest that HCM1 suppresses the expression of YPRO45C. Considering that CLN3 is G1 cyclin and activates $C d c 28$ kinase to promote the $\mathrm{G} 1$ to $\mathrm{S}$ phase transition, we suggest that YPR045C could play a role during G1 and S phase.

\section{Discussion}

Our approach integrates the genetic interaction network, co-expression network, and transcriptional network, and it performed well in predicting cell cycle genes. Many previous papers have also discussing integrating these data source in eQTL analysis. However, comparing to these approaches, we started from known functional genes and their E-MAP profiles to build up the network step by step. In this process, we could see how these data source could describe the biology network, and 

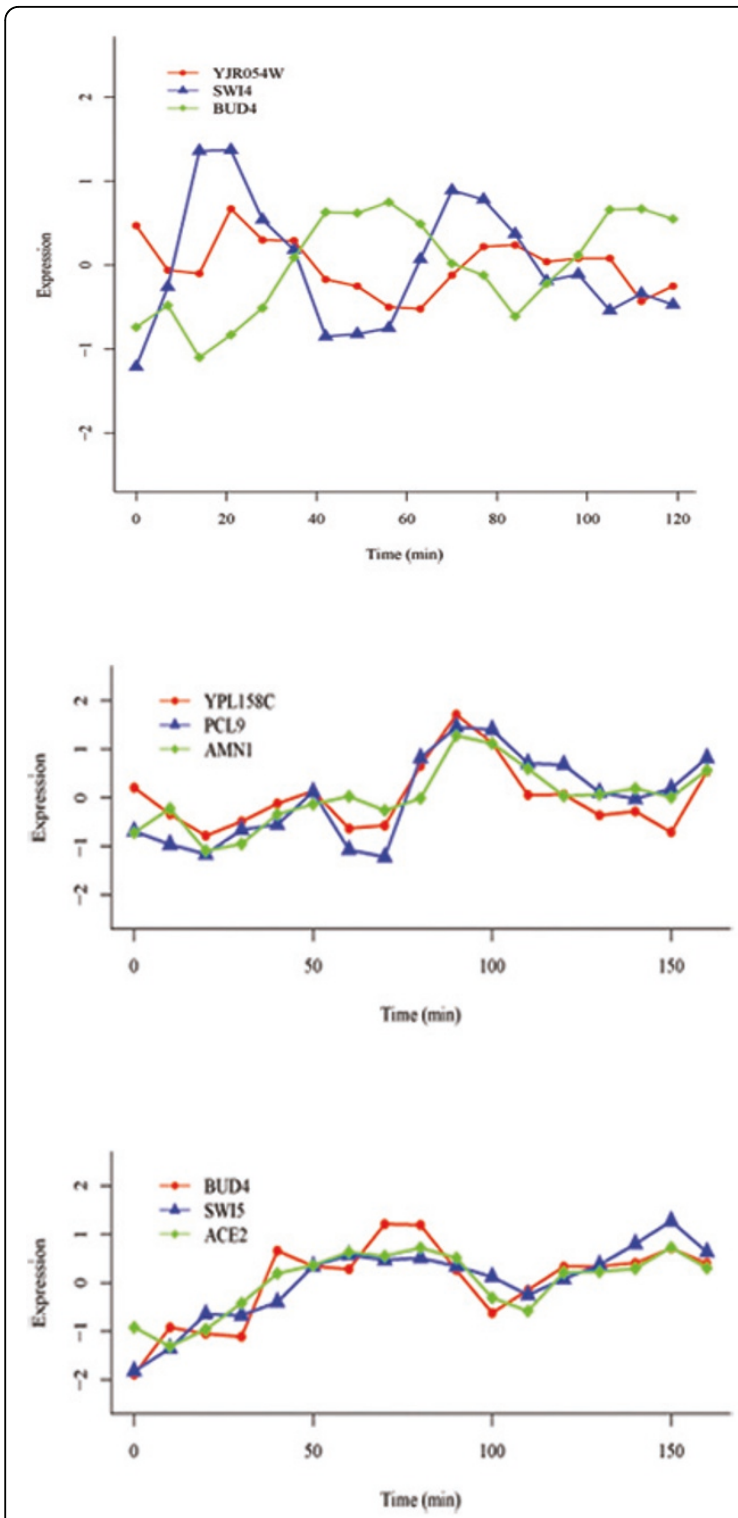
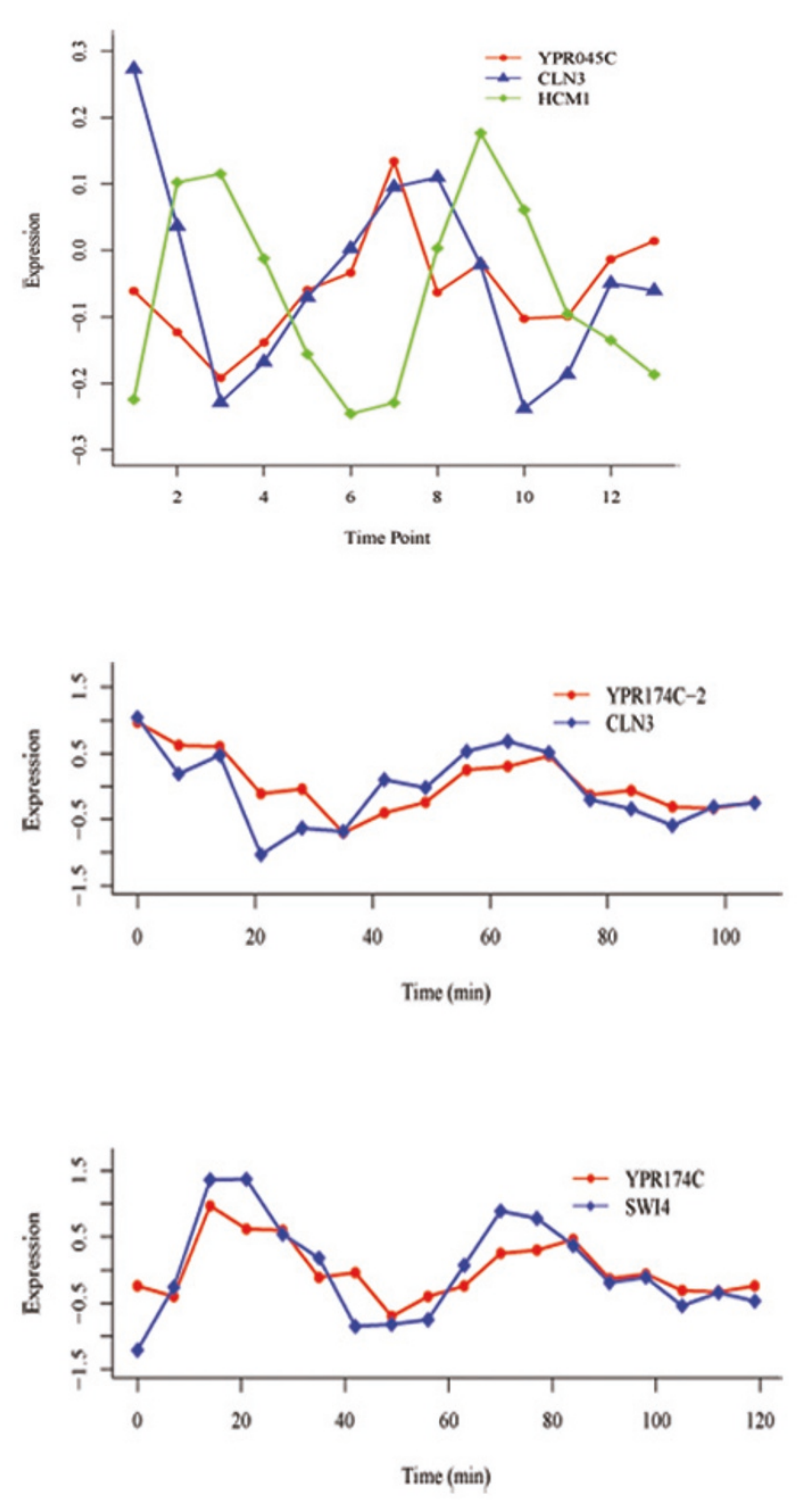

Figure 6 Expression data analysis for the four cell cycle related genes we predicted. (A) The expression of YJR054W, SWI4 and BUD4 in Spellman-alpha experiment. SWI4 and BUD4 are significantly negatively correlated (upper), and YJR054W looks similar to SWI4, although it is appropriately one time point lagged. (B) The expression of YPL158C related genes in Cho-cdc28 experiment. The genes can be separated into two groups. The first group includes YPL158C, PCL9 and AMN1 (upper), and the others form the second group(below). Genes in each group are co-expressed, and the peak value of the first group is lagged comparing to the second one. (C) The expression of YPR174C, CLN3 and SWI4 in Spellman-alpha experiment. YPR174C is co-expressed with SWI4. YPR174C is left shifted for 2 time points (Noted as YPR174C-2), and it's also coexpressed with CLN3. (D) The expression of YPR045C, CLN3 and HCM1 in Pramila-alpha26 experiment. YPR045C and CLN3 are co-expressed, while HCM1 is negative correlated with them and is slightly earlier than them.

how they are co-operated together. We illustrate that EMAP and DNA microarray could be complementary in identifying PCCGs, and also the cluster result tells that how transcriptional relationships could reflect functional connections of genes in the network.

In addition, there are other types of networks, such as protein physical interaction networks, which are informative for the prediction of gene function. However, because physical interactions annotated in databases are quite sparse between KCCGs and the 1536 library strains, we have not performed an analysis of it. We believe the efficiency of prediction can be increased when such data are integrated in a reasonable framework.

Although the current study focused on the cell cycle process, our approach is not limited, and it can be easily 
applied to other biological processes, given the availability of data.

\section{Conclusions}

E-MAP technology is a powerful high-throughput tool to identify novel functional genes which genetic interacted with the known one. By screening forty eight cell cycle genes crossing 1536 library strains, E-MAP helps us obtain a large potential cell cycle-related gene set.

Our analysis shows that genetic interaction and gene co-expression could be complementary for identifying co-functional gene pairs, and combining them has significantly improved the accuracy of the prediction.

TF-DNA binding (chip-chip) and protein phosphorylation data were used to construct a cell cycle regulation network. Periodic expressed and being enriched of cell cycle genes in targets can both be used to identify TFs which regulate the cell cycle process. When comparing the cluster result to prior knowledge, we could believe that our cell cycle transcriptional network is well constructed. This network could help to illustrate how PCCGs are involved in cell cycle process.

Finally, four genes with unknown functions in PCCGs are laboured. From KCCGs which the four genes are genetic interacted and co-expressed, we could predict which phase of cell cycle they may be involved in. In addition, the time course expression data of them are consistent with the constructed transcriptional network, and some of them are substrate of CDK1 (CDC28) kinase which regulates the cell cycle process in budding yeast. All these analyses provided strong evidence that the four novel genes should be participate in cell cycle related process.

\section{Methods and materials \\ E-MAP experiment data}

The 48 cell cycle genes were manually curate from the literature, which function in different phases of the cell cycle process (Figure S5 in Additional file 11). They had been screened by crossing a 1536 mutant strain library in budding yeast, and the relative double mutant strains were selected to obtain the EMAP data. However, the analytical framework we developed is not affected by the selection of these genes, and it can be applied to other processes as well.

Time course expression data and definition of correlation We use eight time course microarray experiments from four previously published works to perform the coexpression analysis [14-17]. The data were downloaded from the supplementary data from the authors' website, and the KNNImpute method was used [18] to impute the missing value.
To measure the similarity between the time course expression profiles of two genes, we used the timelagged correlation [19]. For multiple experiments, we adopted a loose definition for correlation between two genes which is the maximum time lag correlation score in all the eight experiments. This means that two genes showing high correlation in one experiment are considered to be co-expressed. We can use such a loose definition because we have already had a stringent constraint in E-MAP analysis to ensure that the interactions are reliable, even if two genes only show co-expression in one experiment.

\section{Comparison with SGA genetic interaction data}

The true genetic interactions we used here are previously published interactions from Biogrid (http://www. thebiogrid.org). Similar to previous work, for negative genetic interactions, we also considered interactions annotated as phenotypic enhancement, synthetic growth defect and synthetic lethality. For positive genetic interactions, interactions annotated as phenotypic suppression and synthetic rescue are used. By using S-score cut-offs, we calculated the number of true positives (TP) as the number of Biogrid interactions with S-scores more extreme than the cut-offs. As defined in previously published work, sensitivity is defined as the fraction of known interactions.

$$
\text { sensitivity }=\frac{T P}{T P+F N}
$$

Precision is defined as the fraction of true interactions in the set of all predicted pairs.

$$
\text { precision }=\frac{T P}{T P+F P}
$$

We also use hyper geometric distribution to calculate the $\mathrm{p}$-value of precision. The relative results are reported in Table S2 in Additional file 2.

\section{Definition of $p$-values of enrichment analysis for biological process terms in $\mathrm{GO}$}

We also use hyper geometric distribution to calculate pvalue to measure the enrichment of one biological process in $\mathrm{GO}$ annotation as follows:

$$
p_{i}=p\left(m_{i}, n, M_{i}, N\right)=\sum_{k>m_{i}} \frac{C_{N_{i}}^{k} C_{N-M_{i}}^{n-k}}{C_{N}^{n}}
$$

Here $m_{i}$ is the number of selected genes which have the function $\mathrm{i} ; n$ is the number of selected genes; $M_{i}$ is the number of test genes which have the function $\mathrm{i}$; $\mathrm{N}$ is the number of test genes. 
Finally, we use Bonferroni correction to control false discovery rate in this multiple testing problem and get the $\mathrm{q}$ value.

\section{Transcriptional Regulation and CDC28 substrate datasets} The Chip-Chip data and wild type vs. TF mutant microarray data were downloaded from YeastRact (http:// www.yeastract.com[20,21]). Among $183 \mathrm{TFs}$ in our dataset, 37 are annotated as cell cycle-related in the MIPS database. The CDC28 substrate dataset was downloaded from the supplementary data of two previously published works $[22,23]$.

\section{Definition of $p$-values for periodicity and enrichment for cell cycle genes}

The significance of periodicity was previously defined $[24,25]$. The data were downloaded from http://www. cyclebase.org. We also used the hyper geometric distribution to calculate the p-value for the enrichment of cell cycle targets.

$$
p_{i}=p\left(m_{i}, n, M_{i}, N\right)=\sum_{k>m_{i}} \frac{C_{M_{i}}^{k} C_{N-M_{i}}^{n-k}}{C_{N}^{n}}
$$

Here, $m_{i}$ is the neighbor in the PCCGs and KCCGs; $n$ is the number of genes in PCCGs and KCCGs; $M_{i}$ is $T$ $F_{i}$ 's targets in the test genes, and $N$ is the number of test genes.

\section{Multiplication of ranks can represent "or" relationship between the two methods}

Suppose the probability of one $T F_{i}$ not to be enriched and periodically expressed is $p_{i}^{1}, p_{i}^{2}$. Then the probability that $T F_{i}$ is either enriched or periodically expressed is. $\operatorname{Pr}_{i}=1-p_{i}^{1} p_{i}^{2}$. ForTF $F_{i}$, the multiplication of its rank of p-values (ascending order) $r_{i}^{1} r_{i}^{2}$ keeps the order of the probability; $p_{i}^{1} p_{i}^{2}$. Thus the smaller the order of $r_{i}^{1} r_{i}^{2}$ is, the larger $\operatorname{Pr}_{i}$ of $T F_{i}$ is.

\section{Additional material}

Additional file 1: $\mathbf{4 8}$ cell cycle query genes This file can be viewed with Microsoft Excel Viewer.

Additional file 2: Sensitivity and precision of E-MAP genetic interaction scores This file can be viewed with Microsoft Excel Viewer.

Additional file 3: Correlation between our E-MAP data and published data This file can be viewed with Adobe Reader.

Additional file 4: Ranks of p-values for top 25 TFs This file can be viewed with Microsoft Excel Viewer.

Additional file 5: Cover rate when different TFs are selected This file can be viewed with Adobe Reader.

Additional file 6: Cell cycle genes are enriched in the PCCGs and TFs This file can be viewed with Microsoft Excel Viewer.

Additional file 7: CDC28 substrates are enriched in the PCCGs and TFs
Additional file 8: Indirect TF-Target connection analysis This file can be viewed with Adobe Reader.

Additional file 9: Cluster results of transcriptional network This file can be viewed with Adobe Reader. This file can be viewed with Microsoft Excel Viewer.

Additional file 10: Summary of our analytical results This file can be viewed with Microsoft Excel Viewer.

Additional file 11: Composition of the signaling E-MAP This file can be viewed with Adobe Reader.

\section{Acknowledgements}

We thank Hongjing Qu and Xiaojing Yang and Chao Tang lab and Nevan Krogan lab for their help in EMAP experiment. We also thank Dr. David Martin for his critical reading of the manuscript. This work is supported by the National Natural Science Foundation of China (No.10871009, No.10721403, and No.10774009), the National High Technology Research and Development of China (No.2008AA02Z306), the National Key Basic Research Project of China (No. 2006CB910706, No.2009CB918503), and "The Fundamental Research Funds for the Central Universities" in China.

This article has been published as part of BMC Systems Biology Volume 5 Supplement 1, 2011: Selected articles from the 4th International Conference on Computational Systems Biology (ISB 2010). The full contents of the supplement are available online at http://www.biomedcentral.com/17520509/5? issue $=$ S1

\section{Author details}

${ }^{1}$ Center for Theoretical Biology, Peking University, Beijing 100871, China. ¿LMAM, School of Mathematical Sciences, Peking University, Beijing 100871, China. ${ }^{3}$ Center for Statistical Science, Peking University, Beijing 100871, China. ${ }^{4}$ School of Physics, Peking University, Beijing 100871, China.

\section{Authors' contributions}

MD and FTL initiated the study, LW conceived the study and performed the statistical analysis; LH participated in the study and algorithm design and helped to draft the manuscript. All authors read and approved the final manuscript.

\section{Competing interests}

The authors declare that they have no competing interests.

Published: 20 June 2011

\section{References}

1. Mani R, St Onge RP, Hartman JLt, Giaever G, Roth FP: Defining genetic interaction. Proc Natl Acad Sci U S A 2008, 105:3461-3466.

2. Tong $A H$, Evangelista M, Parsons AB, Xu H, Bader GD, Page N, Robinson M, Raghibizadeh S, Hogue CW, Bussey H, et al: Systematic genetic analysis with ordered arrays of yeast deletion mutants. Science 2001, 294:2364-2368.

3. Pan X, Yuan DS, Xiang D, Wang X, Sookhai-Mahadeo S, Bader JS, Hieter P, Spencer $F$, Boeke JD: A robust toolkit for functional profiling of the yeast genome. Mol Cell 2004, 16:487-496.

4. Collins SR, Schuldiner M, Krogan NJ, Weissman JS: A strategy for extracting and analyzing large-scale quantitative epistatic interaction data. Genome Biol 2006, 7:R63-

5. Tu Z, Wang L, Arbeitman MN, Chen T, Sun F: An integrative approach for causal gene identification and gene regulatory pathway inference. Bioinformatics 2006, 22:e489-496.

6. Zhu J, Wiener MC, Zhang C, Fridman A, Minch E, Lum PY, Sachs JR, Schadt EE: Increasing the power to detect causal associations by combining genotypic and expression data in segregating populations. PLoS Comput Biol 2007, 3:e69-.

7. Zhu J, Zhang B, Smith EN, Drees B, Brem RB, Kruglyak L, Bumgarner RE, Schadt EE: Integrating large-scale functional genomic data to dissect the complexity of yeast regulatory networks. Nat Genet 2008, 40:854-861.

8. Lee SI, Dudley AM, Drubin D, Silver PA, Krogan NJ, Pe'er D, Koller D: Learning a prior on regulatory potential from eQTL data. PLOS Genet 2009, 5:e1000358- 
9. Costanzo M, Baryshnikova A, Bellay J, Kim Y, Spear ED, Sevier CS, Ding H, Koh JL, Toufighi K, Mostafavi S, et al: The genetic landscape of a cell. Science 2010, 327:425-431.

10. He X, Qian W, Wang Z, Li Y, Zhang J: Prevalent positive epistasis in Escherichia coli and Saccharomyces cerevisiae metabolic networks. Nat Genet 2010, 42:272-276.

11. Mewes HW, Frishman D, Guldener U, Mannhaupt G, Mayer K, Mokrejs M Morgenstern B, Munsterkotter M, Rudd S, Weil B: MIPS: a database for genomes and protein sequences. Nucleic Acids Res 2002, 30:31-34.

12. Elble R, Tye BK: Both Activation and Repression of a-Mating-Type-Specific Genes in Yeast Require Transcription Factor Mcm1. P Natl Acad Sci USA 1991, 88:10966-10970.

13. Lydall $D$, Ammerer $G$, Nasmyth $K$ : A new role for MCM1 in yeast: cell cycle regulation of SW15 transcription. Genes Dev 1991, 5:2405-2419.

14. Spellman PT, Sherlock G, Zhang MQ, lyer VR, Anders K, Eisen MB, Brown PO, Botstein D, Futcher B: Comprehensive identification of cell cycleregulated genes of the yeast Saccharomyces cerevisiae by microarray hybridization. Mol Biol Cell 1998, 9:3273-3297.

15. Cho RJ, Campbell MJ, Winzeler EA, Steinmetz L, Conway A, Wodicka L, Wolfsberg TG, Gabrielian AE, Landsman D, Lockhart DJ, Davis RW: A genome-wide transcriptional analysis of the mitotic cell cycle. Mol Cell 1998, 2:65-73.

16. de Lichtenberg $U$, Wernersson $R$, Jensen TS, Nielsen HB, Fausboll A, Schmidt $P$, Hansen FB, Knudsen S, Brunak S: New weakly expressed cell cycle-regulated genes in yeast. Yeast 2005, 22:1191-1201.

17. Pramila T, Wu W, Miles S, Noble WS, Breeden LL: The Forkhead transcription factor $\mathrm{Hcm} 1$ regulates chromosome segregation genes and fills the S-phase gap in the transcriptional circuitry of the cell cycle. Genes Dev 2006, 20:2266-2278.

18. Troyanskaya O, Cantor M, Sherlock G, Brown P, Hastie T, Tibshirani R, Botstein D, Altman RB: Missing value estimation methods for DNA microarrays. Bioinformatics 2001, 17:520-525.

19. Bushel PR, Heard NA, Gutman R, Liu L, Peddada SD, Pyne S: Dissecting the fission yeast regulatory network reveals phase-specific control elements of its cell cycle. BMC Syst Biol 2009, 3:93-

20. Teixeira MC, Monteiro $P$, Jain $P$, Tenreiro S, Fernandes AR, Mira NP Alenquer M, Freitas AT, Oliveira AL, Sa-Correia I: The YEASTRACT database: a tool for the analysis of transcription regulatory associations in Saccharomyces cerevisiae. Nucleic Acids Res 2006, 34:D446-451

21. Monteiro PT, Mendes ND, Teixeira MC, d'Orey S, Tenreiro S, Mira NP, Pais $H$, Francisco AP, Carvalho AM, Lourenco AB, et al: YEASTRACT-DISCOVERER: new tools to improve the analysis of transcriptional regulatory associations in Saccharomyces cerevisiae. Nucleic Acids Res 2008, 36 D132-136.

22. Ubersax JA, Woodbury EL, Quang PN, Paraz M, Blethrow JD, Shah K, Shokat KM, Morgan DO: Targets of the cyclin-dependent kinase Cdk1. Nature 2003, 425:859-864.

23. Holt LJ, Tuch BB, Villen J, Johnson AD, Gygi SP, Morgan DO: Global Analysis of Cdk1 Substrate Phosphorylation Sites Provides Insights into Evolution. Science 2009, 325:1682-1686.

24. Gauthier NP, Jensen L, Wernersson R, Brunak S, Jensen TS: Cyclebase.org: version 2.0, an updated comprehensive, multi-species repository of cell cycle experiments and derived analysis results. Nucleic Acids Res 2010, 38 : D699-702.

25. Gauthier NP, Larsen ME, Wernersson R, de Lichtenberg U, Jensen LJ, Brunak S, Jensen TS: Cyclebase.org-a comprehensive multi-organism online database of cell-cycle experiments. Nucleic Acids Res 2008, 36: D854-859.

doi:10.1186/1752-0509-5-S1-S9

Cite this article as: Wang et al:: Integrating multiple types of data to predict novel cell cycle-related genes. BMC Systems Biology 2011 5(Suppl 1):S9.

\section{Submit your next manuscript to BioMed Central and take full advantage of:}

- Convenient online submission

- Thorough peer review

- No space constraints or color figure charges

- Immediate publication on acceptance

- Inclusion in PubMed, CAS, Scopus and Google Scholar

- Research which is freely available for redistribution 